\title{
Konstituierung von Kleinunternehmer-Selbsthilfegruppen im städtischen Java, Indonesien ${ }^{1}$
}

In- und ausländische Entwicklungsanstrengungen in Drittweltländern konzentrierten sich in den beiden Dekaden der Nachkriegszeit auf technische und physischmaterielle Elemente, wie z.B. die Verbesserung und Transferierung von Technologien oder den Aufbau von Infrastruktur. Erst in den siebziger Jahren wurden auch institutionelle und organisationelle Aspekte berücksichtigt und beispielsweise in Zusammenarbeit mit staatlichen Organisationen Genossenschaften aufgebaut und gefördert. Seit den achtziger Jahren werden solche «Software»-Aspekte immer mehr als zentraler Bestandteil der Entwicklung perzipiert.

Gleichzeitig zum oben erwähnten Trend wird auch die Zweckmäßigkeit und die Effizienz staatlicher Organisationen hinsichtlich der Verbesserung der Lebenssituation armer Bevölkerungsschichten in Entwicklungsländern von verschiedenen Seiten angezweifelt. Statt dessen muten Entwicklungstheoretiker wie -praktiker der " $\mathrm{Zi}$ vilgesellschaft» vermehrt ein großes Entwicklungspotential zu. ${ }^{2}$ Dies zeigt sich z. B. in einem verstärkten Vertrauen in die Selbsthilfe der lokalen Bevölkerung und in einer wachsenden Hinwendung von Entwicklungshilfegebern aus den Industrieländern zu nichtstaatlichen Entwicklungsorganisationen $($ NGOs $=$ «Non-Governmental Organizations») der Dritten Welt.

Die verstärkte Berücksichtigung von institutionellen Aspekten und von NGOs führt dazu, daß Entwicklungsorganisationen häufig nach bestehenden, weder staatlichen noch formellen marktwirtschaftlichen Organisations- und Kooperationsformen unter Personen in ähnlicher sozioökonomischer Situation suchen und/oder daß lokale, vom Staat unabhängige Organisationen aus der Projektzielbevölkerung aufgebaut und gefördert werden. Im Zusammenhang mit der Umorientierung von nachholender zu nachhaltiger Entwicklung ${ }^{3}$ gewinnen institutionelle und organisationelle Aspekte der Entwicklung auf der Projektebene an Bedeutung: Die Zielgruppen selbst sollen nach Ablauf der eigentlichen Unterstützungsphasen zu Trägerorganisationen von Projekten werden und diese nachhaltig weiterführen. Solche $\mathrm{Zu}$ sammenschlüsse aus (meist erwerbsspezifischen) Segmenten der lokalen Bevölkerung werden im Entwicklungsjargon Selbsthilfegruppen genannt.

Vor diesem Hintergrund wächst das Interesse an möglichen Erklärungen für das erfolgreiche Entstehen und Fortbestehen von Selbsthilfegruppen. In der Literatur wurden bis anhin vor allem landesspezifische politische und ökonomische Bedingungen für das Entstehen von
Selbsthilfe oder Möglichkeiten und Grenzen von inländischen NGOs im Hinblick auf die Förderung von Selbsthilfegruppen thematisiert. ${ }^{4}$ Für einen weiteren Beitrag zur Erklärung des Erfolges von Selbsthilfegruppen sollen in diesem Artikel auch die gruppeninternen Prozesse und Strukturen berücksichtigt und zu den Rahmenbedingungen in Bezug gesetzt werden. Am Beispiel von Kleinunternehmer-Selbsthilfegruppen des mechanisierten Kleingewerbes im städtischen Java, Indonesien, sollen solche gruppenexterne und -interne Kräfte sowie deren Zusammenspiel diskutiert werden. ${ }^{5}$

\section{Kleinunternehmer-Selbsthilfegruppen des mechanisierten Kleingewerbes im städtischen Java}

108 Mio. von insgesamt 180 Mio. Einwohnern und Einwohnerinnen Indonesiens leben auf der Insel Java, welche weniger als $7 \%$ der Landfläche des indonesischen Archipels einnimmt. Die «einheimische» Bevölkerung Javas läßt sich grob in die Sundanesen in Westjava und die Javaner im restlichen Teil der Insel unterteilen (vgl. Karte in Abb. 1). So wird denn nebst der offiziellen Nationalsprache Bahasa Indonesia in Westjava auch heute noch vorwiegend sundanesisch und im restlichen Java meist javanisch gesprochen (vgl. LEEMANN 1993: 4).

Zwischen 1980 und 1990 wuchs die indonesische Bevölkerung um 1,8\% pro Jahr. Gleichzeitig ist der Anteil der städtischen Bevölkerung im Steigen begriffen; heute beträgt er $31 \%$. So wächst denn auch die Bedeutung des sekundären und des tertiären Sektors, u. a. auch des städtischen Kleingewerbes. ${ }^{6}$ Nebst traditionellem Handwerk bestehen im städtischen Kleingewerbe auch Betriebe, welche für die Güterproduktion Maschinen einsetzen. Für letztere wird hier der Begriff «mechanisiertes Kleingewerbe» verwendet. Betriebe im Kleingewerbe werden als «Kleinunternehmen» bezeichnet und als Einheiten mit höchstens 20 Angestellten, in denen der Besitzer selbst im Betriebsmanagement tätig ist, definiert.

Kleinunternehmer-Selbsthilfegruppen ${ }^{7}$ bestehen aus Besitzern/Betriebsleitern von Kleinunternehmen. Meine Definition solcher Gruppen umfaßt folgende vier Punk-

René Véron, dipl. Geograph, Geographisches Institut der Universität Zürich Irchel, Winterthurerstraße 190, 8057 Zürich 


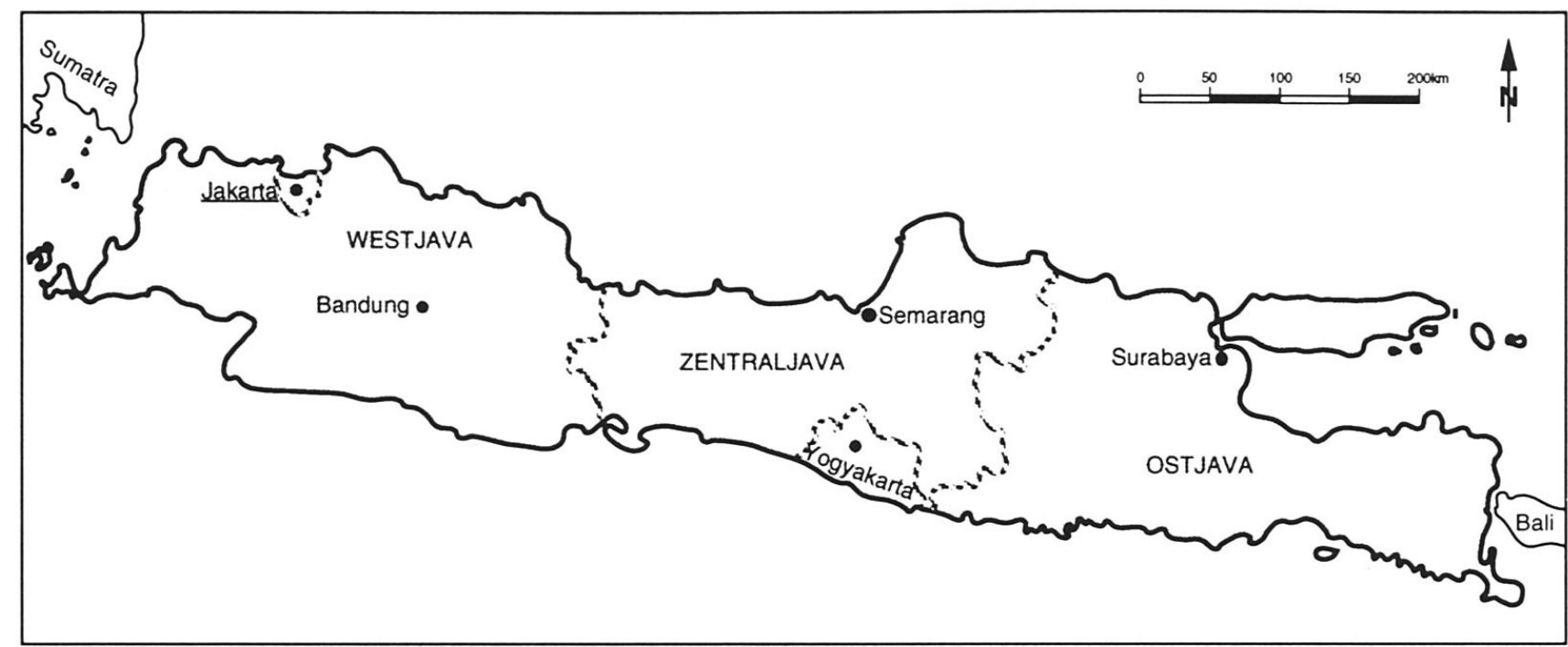

Abb.1 Übersichtskarte von Java

te. Erstens ist eine Kleinunternehmer-Selbsthilfegruppe eine freiwillige Vereinigung von Kleinunternehmern, welche durch mindestens ein gemeinsames Ziel verbunden sind. Zweitens beabsichtigen die Mitglieder, mittels der Vereinigung ihre soziale und/oder wirtschaftliche Lage zu verbessern. Drittens sind die Mitglieder Empfänger der Vorteile, die durch gemeinsame Aktivitäten hervorgebracht werden, wobei diese durch Leistungen einer professionellen Leitung oder durch externe Unterstützung ergänzt werden können. Viertens weist der Entscheidungsfindungsproze $\beta$ innerhalb einer Selbsthilfegruppe insofern partizipative Elemente auf, als die gewöhnlichen Mitglieder die Möglichkeit haben, über (egalitäre oder hierarchische) Organisationsstrukturen zu entscheiden. Die untersuchten Vereinigungen sind berufsgruppenspezifische Selbsthilfegruppen, d.h., die Mitglieder arbeiten in der gleichen Branche.

Die Felduntersuchungen im städtischen Java haben gezeigt, daß nur eine kleine Minderheit der Kleinunternehmer in aktiven Selbsthilfegruppen organisiert sind. Im mechanisierten Kleingewerbe sind weder traditionelle noch neuere, vollkommen autonom entstandene Organisations- oder Kooperationsformen erkennbar. ${ }^{8}$ Vielmehr haben sich Kleinunternehmer-Selbsthilfegruppen in direktem oder indirektem Zusammenhang mit einem extern initiierten Förderungsprojekt entwickelt. ${ }^{9}$

Die 15 erfaßten Selbsthilfegruppen unterscheiden sich hauptsächlich bezüglich der Fortgeschrittenheit ihres Gruppenbildungsprozesses und hinsichtlich ihrer Aktivitätsbereiche. In einer Anfangsphase haben neue, meist von einer Entwicklungsorganisation initiierte Selbsthilfegruppen (noch) brüchige interne Strukturen und führen erst in beschränktem Umfang Aktivitäten durch. Solche Selbsthilfegruppen dienen meistens nur dem Austausch und der Weitergabe von Informationen. Zum Teil werden Meetings zu nicht näher festgelegten Themen ab- gehalten. Selbsthilfegruppen in einer Aufbauphase weisen bereits gefestigtere Strukturen und - neben häufig durchgeführten Spar/Leih-Aktivitäten unter den Mitgliedern - normalerweise nur eine Hauptaktivität auf, entweder im Bereich der Vermarktung, der Produktion oder der Interessenvertretung. Weitere Aktivitäten sind eventuell im Aufbau, aber spielen (noch) eine unbedeutende Rolle. Selbsthilfegruppen in einem konsolidierten Stadium zeichnen sich durch ein vielfältiges und ausgewogenes Leistungsangebot und durch größere Unabhängigkeit von unterstützenden Entwicklungsorganisationen aus. Bei fehlender Initiative und ausbleibendem gruppenbezogenem Handeln der Mitglieder aber können Selbsthilfegruppen in ein Ruhestadium fallen und ihre Aktivitäten (zeitweise) beenden. Die beschriebenen Phasen müssen von einer Selbsthilfegruppe nicht zwingend nacheinander durchlaufen werden. Zudem sind die Übergänge fließend und die Abgrenzung nicht immer eindeutig.

Selbsthilfegruppen, die im Vermarktungsbereich aktiv sind, kaufen Produkte ihrer Mitglieder auf und vermarkten diese an Händler, staatliche Kooperativen, Großunternehmen usw. Die gemeinsame Vermarktung erschließt für Kleinunternehmer häufig neue (selten auch internationale) Märkte und ist zum Teil mit technischen Trainings, Diffusion neuer Produktedesigns, Animation zu Innovationen oder Preis- und Qualitätsstandardisierungen verbunden. Ein Beispiel einer VermarktungsSelbsthilfegruppe ist die Koperasi Industri Batur Jaya ( = staatliche Industriegenossenschaft Batur Jaya), in der 145 Gießereien aus der Umgebung der zentraljavanischen Mittelstadt Klaten zusammengeschlossen sind. In dieser Gruppe werden hauptsächlich Aufträge vom Staat oder von Großunternehmen organisiert und unter den Mitgliedern aufgeteilt. 
Die Zusammenarbeit von Kleinunternehmern im Produktionsbereich beinhaltet meistens die Aufteilung von Arbeitsschritten gemäß individueller Spezialisierung der Gruppenmitglieder. Das Produkt wird in der Regel auch gemeinsam vermarktet. Mit solchen Kooperationen können zum Teil sogar neue Produktionsnischen für das Kleingewerbe erschlossen werden, ohne daß andere Kleinunternehmen verdrängt würden. So produzieren beispielsweise zwölf Kleinunternehmer der Metallbranche in der Stadt Tegal, welche in der Kelompok Produksi Mesin (= Maschinenproduktionsgruppe) zusammengeschlossen sind, in Lizenz für eine Großfirma Handtraktoren, was ohne Selbsthilfegruppe und ohne Aufteilung der notwendigen Arbeitsschritte für ein einzelnes Kleinunternehmen nicht möglich wäre.

Im Bereich der Interessenvertretung arbeitende Selbsthilfegruppen setzen sich für Anliegen ihrer Mitglieder bei (v. a. lokalen) Behörden, bei Banken, bei Großunternehmen usw. ein. In einer Gruppe vereint und eventuell von einer Entwicklungsorganisation unterstützt, können Rechtssicherheiten für Kleinunternehmer oder infrastrukturelle Verbesserungen leichter durchgesetzt werden, und umgekehrt kann Verschlechterungen besser entgegengewirkt werden.

Ziel der von Selbsthilfegruppen häufig durchgeführten Spar/Leih-Aktivitäten (simpan pinjam) ist, die Mitglieder zur Kapitalbildung anzuhalten und ihnen die Möglichkeit zu verschaffen, bei Bedarf Kredite zu günstigen Konditionen aus gemeinsamen Gruppenersparnissen aufzunehmen. Denn wirtschaftlich Schwache haben aufgrund fehlender Rücklagen kaum Zugang zu formalen Bankkrediten, so daß sie zur Mittelbeschaffung häufig auf informelle, oft Wucherzinse verlangende Geldverleiher angewiesen sind. Letztere können dank Spar/LeihGruppen umgangen werden. Simpan pinjam funktioniert vor allem dann gut, wenn die soziale Kontrolle unter den Gruppenmitgliedern ausgeprägt ist. Diese Spar/LeihGruppen beruhen auf einer in Indonesien kulturell verankerten Institution - dem $\operatorname{arisan}^{10}$

\section{Rahmenbedingungen für die Selbsthilfe im städtischen Kleingewerbe}

Die wirtschaftliche Situation im mechanisierten Kleinbetriebssektor präsentiert sich von Branche zu Branche, von Region zu Region und von Einzelbetrieb zu Einzelbetrieb äußerst unterschiedlich und ist auch globalen, nationalen und regionalen wirtschaftlichen Entwicklungen unterworfen. Die allgemeinen wirtschaftspolitischen Rahmenbedingungen in Indonesien sind für Kleinunternehmen eher ungünstig und können nicht durch die speziellen staatlichen Kleingewerbeförderungsprogramme kompensiert werden (vgl. HILL 1992). Somit ist der Aufbau von Kleinunternehmer-Selbsthilfegruppen bzw. das Zusammenlegen vorhandener Ressourcen der Kleinunternehmer (technische und unternehmerische Fähigkei- ten, betriebliche Ausstattung, Kapital) unter etlichen branchen-, regions- und betriebsspezifischen Voraussetzungen aus wirtschaftlicher Sicht sinnvoll.

Soziokulturelle Hintergründe erschweren jedoch ein spontanes Entstehen von gruppenbezogener Selbsthilfe außerhalb des familiären Bereichs unter sozial Gleichgestellten der Unterschicht oder der unteren Mittelschicht, wozu Kleinunternehmer in der Regel gehören. In der hierarchisch strukturierten javanischen bzw. sundanesischen (= westjavanischen) Gesellschaft haben vertikale Beziehungen, traditionell in Form von personenbezogenen Patron-Klientel-Beziehungen, ihre dominante Bedeutung bis heute insbesondere als wirtschaftliche Abhängigkeitsverhältnisse behalten. Dieser Umstand hemmt die Initiative zu gruppenbezogener Selbsthilfe unter Kleinunternehmern. Schwächer ausgeprägte horizontale Beziehungen zwischen Personen gleichen (niedrigen) sozialen Status wie die Nachbarschaftshilfe (tolong-menolong) lösen sich vor allem in städtischen Gebieten im Zuge des sozialen Wandels langsam auf (MULDER 1990: 88 ff.). Es ist mir denn auch kein aktueller Fall im städtischen mechanisierten Kleingewerbe bekannt, bei dem tolong-menolong auf eine wirtschaftliche Zusammenarbeit zwischen Kleinunternehmern ausgedehnt worden wäre."

Gerade in städtischen Gebieten ist aber ein Trend zu neuen horizontalen Beziehungen feststellbar, die auf gemeinsamen Interessen zwischen sozial Gleichgestellten wie Arbeitskollegen und -kolleginnen oder Bekannten beruhen und nicht an die nachbarschaftliche Gemeinschaft gebunden sind (MULDER 1990: 88 ff.). Solche Beziehungen können eine geeignete Basis für den Aufbau von Kleinunternehmer-Selbsthilfegruppen darstellen. Wegen der nach wie vor dominanten Rolle vertikaler Beziehungen zwischen Kleinunternehmern und Zwischenhändlern oder Großunternehmen, welche von Kleinunternehmen produzierte Halbfabrikate aufkaufen, können sich solche neuartigen horizontalen Beziehungen jedoch kaum ohne externe Unterstützung entfalten.

Zusätzlich zu diesen hinsichtlich des Entstehens von Selbsthilfe eher ungünstigen allgemeinen soziokulturellen Voraussetzungen kommen noch hinderliche spezielle Merkmale im städtischen mechanisierten Kleingewerbe hinzu. Dazu gehören ein Mangel an traditionellen Kooperationsformen und etablierten Kontakten zwischen Berufskollegen gleicher Branche, eine im allgemeinen eher individualistische Einstellung der Kleinunternehmer und zum Teil negative Erfahrungen von Kleinunternehmern mit erfolglosen staatlichen Genossenschaften. All dies führt dazu, daß die Bildung von Kleinunternehmer-Selbsthilfegruppen in der Regel schwierig ist oder zumindest eine längere Förderungsphase voraussetzt. Letzteres bedeutet, daß NGOs, welche die wichtigsten Förderer von Selbsthilfe sind und in Indonesien Lembaga Pengembangan Swadaya Masyarakat (= Selbsthilfeförderungsorganisation) genannt werden, eine zentrale Rolle im Umfeld von Kleinunternehmer-Selbsthilfegruppen einnehmen. Vor allem bei der Interessenvertretung gegenüber Behörden, Banken, Zwischenhändlern oder 
Großunternehmen bedürfen Selbsthilfegruppen langfristigen Beistands. Dieser Umstand trägt dazu bei, daß auch zwischen NGOs und ihren Selbsthilfegruppen Abhängigkeitsbeziehungen entstehen können. Solche Beziehungen tragen oft Charaktereigenschaften traditioneller Patron-Klientel-Beziehungen; traditionelle javanische Werte werden durch größere Jakarta-basierte NGOs reproduziert (FRINGS 1991). Ein zumindest ebenso wichtiger Grund, daß die Tätigkeiten von NGOs in Indonesien nicht in demokratischen sozialen Bewegungen resultieren, liegt in der Tatsache, daß die Handlungsspielräume der NGOs durch staatliche Kontrolle stark eingeschränkt werden. Der Staat toleriert keine Form der Massenmobilisierung, welche dessen Macht untergraben könnte (vgl. ELDRIDGE 1989). So stoßen NGOs und Selbsthilfegruppen vor allem bei lokalen Beamten häufig auf Mißtrauen und Ablehnung (HOLLOWAY, 1989).

Neben den in Indonesien allgemein geltenden Rahmenbedingungen existiert für jede Kleinunternehmer-Selbsthilfegruppe auch ein spezifisches Umfeld in Form der unterstützenden Entwicklungsorganisation und ihrem angewandten Förderungsansatz sowie in Form lokal- und branchenspezifischer Gegebenheiten wie Produktionsund Vermarktungsstrukturen oder Entscheide und Einstellungen lokaler Behörden. Weiter unten wird am Beispiel einer Schreiner-Selbsthilfegruppe ein solches Umfeld dargestellt.

\section{Konstituierungsprozeß von Selbsthilfegruppen durch das Handeln von Kleinunternehmern}

Ein zentrales Resultat der Untersuchungen ist, daß die Handlungen der Kleinunternehmer und gruppeninterne Faktoren mindestens ebenso wesentlich für die Konstituierung von Selbsthilfegruppen sind wie gruppenexterne Faktoren (allgemeine Rahmenbedingungen, spezifisches Umfeld). Selbsthilfegruppen sind nicht primär Produkte ihrer Umgebung, denn unter ähnlichen gruppenexternen Faktoren sind nicht überall Selbsthilfegruppen entstanden, und umgekehrt gibt es Selbsthilfegruppen, die in unterschiedlichen Umfeldern erfolgreich funktionieren. Letztlich werden Selbsthilfegruppen durch das Handeln ihrer Mitglieder konstituiert und können ohne deren Willen niemals entstehen. Dabei sind gruppenexterne Faktoren nicht irrelevant, sondern sie wirken als Handlungsbedingungen, welche individuelle Handlungsorientierungen und -ziele mitbeeinflussen können, auf die Kleinunternehmer.

Aus den Untersuchungen im städtischen mechanisierten Kleingewerbe auf Java gehen vier gruppeninterne Faktoren hervor, welche wesentliche Handlungsbedingungen und -folgen für die Konstituierung von Selbsthilfegruppen darstellen. Die vier wichtigen Faktoren sind (vgl. VERON 1993: 108 ff.):

- Gemeinsamkeiten in individuellen Interessen,

- Führungspersönlichkeiten und Mitgliederpartizipation,

- Zusammengehörigkeitsgefühl,
- individueller ökonomischer Nutzen

durch die Selbsthilfegruppe.

Bei der Bildung von Kleinunternehmer-Selbsthilfegruppen besteht dann eine günstige Ausgangssituation, wenn Gemeinsamkeiten in den individuellen Interessen unter den potentiellen Gruppenmitgliedern auftreten. Das Vorhandensein von starken, engagierten und integren Führungspersönlichkeiten und aktiven Mitgliedern sowie ein starkes Zusammengehörigkeitsgefühl in der Gruppe sind wichtige Prinzipien günstiger sozialer Beziehungen unter den (potentiellen) Mitgliedern. Unter diesen Bedingungen besteht die Chance, daß eine Selbsthilfegruppe entsteht, die in der Lage ist, mitgliedergerechte Leistungen hervorzubringen. Wenn diese Leistungen den Kleinunternehmern in der Folge individuellen ökonomischen Nutzen bringen, dann haben die Kleinunternehmer auch ein besonderes Interesse, weiterhin aktiv in der Selbsthilfegruppe zu sein. Es können so neue Gemeinsamkeiten in individuellen Interessen erkannt werden, und das Zusammengehörigkeitsgefühl unter den Kleinunternehmern kann sich weiter verstärken. Auf diese Weise verbessert sich die Ausgangssituation wiederum, und die Chance erhöht sich, daß die Selbsthilfegruppe durch fortwährendes gruppenbezogenes Handeln der Kleinunternehmer reproduziert wird und fortbesteht.

\section{Die vier gruppeninternen Faktoren \\ für die Konstituierung \\ von Kleinunternehmer-Selbsthilfegruppen}

\section{Gemeinsamkeiten in individuellen Interessen}

Unter Mitgliedern einer Selbsthilfegruppe muß Konsens über mindestens ein anzustrebendes, aus einem gemeinsamen Interesse abgeleitetes Ziel herrschen. Gemeinsame Interessen werden beispielsweise dann relativ leicht erkannt, wenn sich alle Mitglieder durch einen Druck gleichermaßen betroffen fühlen. Dadurch wird auch der Gruppenzusammenhalt gestärkt, und die Kleinunternehmer sind eher bereit, untereinander zu kooperieren. Bei den meisten Kleinunternehmer-Selbsthilfegruppen bestand die Gemeinsamkeit in den individuellen Interessen der Gründungsmitglieder darin, die Konkurrenz und den Preiskrieg untereinander zu beenden bzw. zu lindern. Dieser Wille beruht auf der Einsicht, daß ein unbarmherziger Konkurrenzkampf unter Kleinunternehmen langfristig zur Selbstzerstörung führen würde, insbesondere dann, wenn man zusätzlich dem harten Wettbewerb seitens der Großunternehmen und Zwischenhändler ausgesetzt ist. Divergieren umgekehrt die Absichten bezüglich der Partizipation in einer Selbsthilfegruppe, werden vermehrt Partikularinteressen verfolgt, was eine erfolgreiche Tätigkeit erschwert.

\section{Zusammengehörigkeitsgefühl}

Ein ausgeprägtes Zusammengehörigkeitsgefühl ist vor allem deshalb bedeutsam, weil die Mitgliedschaft in einer 
Selbsthilfegruppe auf Freiwilligkeit basiert und kein starker Zwang ausgeübt werden kann. Nur wenn ein gut entwickeltes Zusammengehörigkeitsgefühl besteht und sich die Mitglieder mit der Gruppe identifizieren, werden sie sich auch aktiv darin engagieren, was Voraussetzung für die Konstituierung einer Selbsthilfegruppe ist. Die Entwicklung eines Zusammengehörigkeitsgefühls gestaltet sich bei Kleinunternehmern besonders schwierig, weil sie primär Konkurrenten sind und in der Regel individualistisch handeln. Wenn dennoch die Einsicht entsteht, daß sich die Berufskollegen in derselben (mißlichen) Situation befinden, kann sich aus gemeinsamen Interessen und durch erste Gruppenaktivitäten langsam eine affektuale Zusammengehörigkeit entwickeln. Bevor mit größeren gemeinsamen Aktivitäten begonnen werden kann, muß sich ein vertrauensbildender Prozeß abspielen.

\section{Führungspersönlichkeiten und Mitgliederpartizipation}

Die Untersuchungen zeigten, daß auch das Vorhandensein integrer, engagierter und starker Führungspersönlichkeiten sowie aktiver Mitglieder zur Konstituierung einer Selbsthilfegruppe wichtig ist. Die herausragende Bedeutung geeigneter Führungspersönlichkeiten rührt zum Teil von den soziokulturellen Rahmenbedingungen her. Organisationen mit einer starken Führungspersönlichkeit sind in der hierarchisch strukturierten indonesischen Gesellschaft tendenziell erfolgreicher, denn die meisten Indonesier und Indonesierinnen orientieren sich stark an Leitfiguren. Die Rolle des Leiters in einer Selbsthilfegruppe ist deshalb ausschlaggebender als bei genossenschaftlichen Gruppen in Europa. Engagierte, starke Führungspersönlichkeiten sollten dank Sachverstand und gemeinnützigem Handeln (und nicht nur durch einen vorgegebenen formellen Status) in eine Machtposition gelangen. Solche Personen genießen hohes Ansehen und Vertrauen und wirken integrierend. Damit aber auch sichergestellt werden kann, daß mitgliedergerechte Aktivitäten durchgeführt werden, müssen die Formulierung von Zielen und die Wahl der Leitung in der Kompetenz der Mitglieder liegen. Wenn in einer Selbsthilfegruppe partizipative Strukturen fehlen, fühlen sich die Beteiligten ohnmächtig, schlecht repräsentiert und werden passiv oder kündigen die Mitgliedschaft. Ohne aktive Mitglieder, die auch die Führung kontrollieren, zerfällt eine Selbsthilfegruppe zu einem organisatorischen Gefäß ohne Inhalt bzw. ohne Aktivitäten.

\section{Individueller ökonomischer Nutzen durch die Selbsthilfegruppe}

Gemeinsamkeiten in individuellen Interessen, Zusammengehörigkeitsgefühl sowie Führungspersönlichkeiten und Mitgliederpartizipation sind dafür entscheidend, ob sich als Folge der Handlungen überhaupt eine Selbsthilfegruppe bildet, die imstande ist, für ihre Mitglieder angepaßte Leistungen zu erbringen. Für das Fortbestehen bzw. die Reproduktion einer Selbsthilfegruppe müssen für die einzelnen Akteure zusätzlich positive Effekte aus der aktiven Mitgliedschaft in einer Selbsthilfegruppe erwachsen. Die Untersuchungen lassen erkennen, daß es den Kleinunternehmern hauptsächlich darum geht, aus der Mitgliedschaft in einer Selbsthilfegruppe wirtschaftlichen Nutzen für den eigenen Betrieb ziehen zu können. Das Erwachsen eines individuellen ökonomischen Nutzens dank den Aktivitäten einer Selbsthilfegruppe führt auch zu steigendem Interesse am Zusammenschluß. Kleinunternehmer mögen dann weitere Interessengemeinsamkeiten erkennen und haben dank positiven Erfahrungen Mut zu neuen gemeinsamen Aktivitäten. Zudem wird das Zusammengehörigkeitsgefühl verstärkt. Diese Rückkoppelungseffekte tragen zur erfolgreichen Reproduktion bzw. Nachhaltigkeit einer Selbsthilfegruppe bei. Wenn sich für die Kleinunternehmer hingegen keine positiven Handlungsfolgen einstellen, läuft eine Selbsthilfegruppe Gefahr, sich aufzulösen. Der Erfolg und das Fortbestehen einer Selbsthilfegruppe hängen somit in hohem Maß davon ab, ob die hervorgebrachten Dienstleistungen den Kleinunternehmern wirtschaftliche Vorteile erbringen oder nicht. In welchen Bereichen die Dienstleistungen einer Selbsthilfegruppe sinnvoll durchführbar sind, hängt von der speziellen wirtschaftlichen Situation und den spezifischen gemeinsamen Interessen der Gruppenmitglieder ab. Es gilt lediglich das Prinzip, daß sich die Aktivitäten stets den sich ändernden Bedingungen, Bedürfnissen und Interessen der Kleinunternehmer anpassen müssen, so daß diesen ein Nutzenvorteil zukommt.

Bevor anhand eines Fallbeispiels die gegenseitige Beeinflussung des Umfelds einer Selbsthilfegruppe und der gruppeninternen Prozesse veranschaulicht wird, sind in Abb. 2 die identifizierten relevanten gruppenexternen und -internen Faktoren für die Konstitution von Kleinunternehmer-Selbsthilfegruppen zusammengefaßt.

\section{Fallbeispiel: \\ Schreiner-Selbsthilfegruppe $K I P K A B$ in Bandung}

Bandung ist Hauptstadt der Provinz Westjava und mit rund zwei Millionen Einwohnern und Einwohnerinnen drittgrößte Stadt Indonesiens (NUDS 1985). ${ }^{12}$ Nach dem Großraum Jakarta und der ostjavanischen Hafenstadt Surabaya gehört Bandung auch zu den am meisten industrialisierten Gebieten Indonesiens. Nebst der Großindustrie (vor allem Textil-, aber auch chemische und Flugzeugbauindustrie) existieren in Bandung eine Vielzahl von Werkstätten im verarbeitenden mechanisierten Kleingewerbe (vor allem Nahrungsmittel-, Textil/Leder-, Holz-, Metallverarbeitung und Baustoffherstellung) (SChMidT 1988: 94). Das folgende Beispiel einer Kleinunternehmer-Selbsthilfegruppe stammt aus dem holzverarbeitenden Kleingewerbe.

Die Mehrzahl der Schreinereien in Bandung sind entlang einer Hauptstraße im Süden der Stadt Bandung angesiedelt, wo sich Betrieb an Betrieb reiht. Sie produzieren 


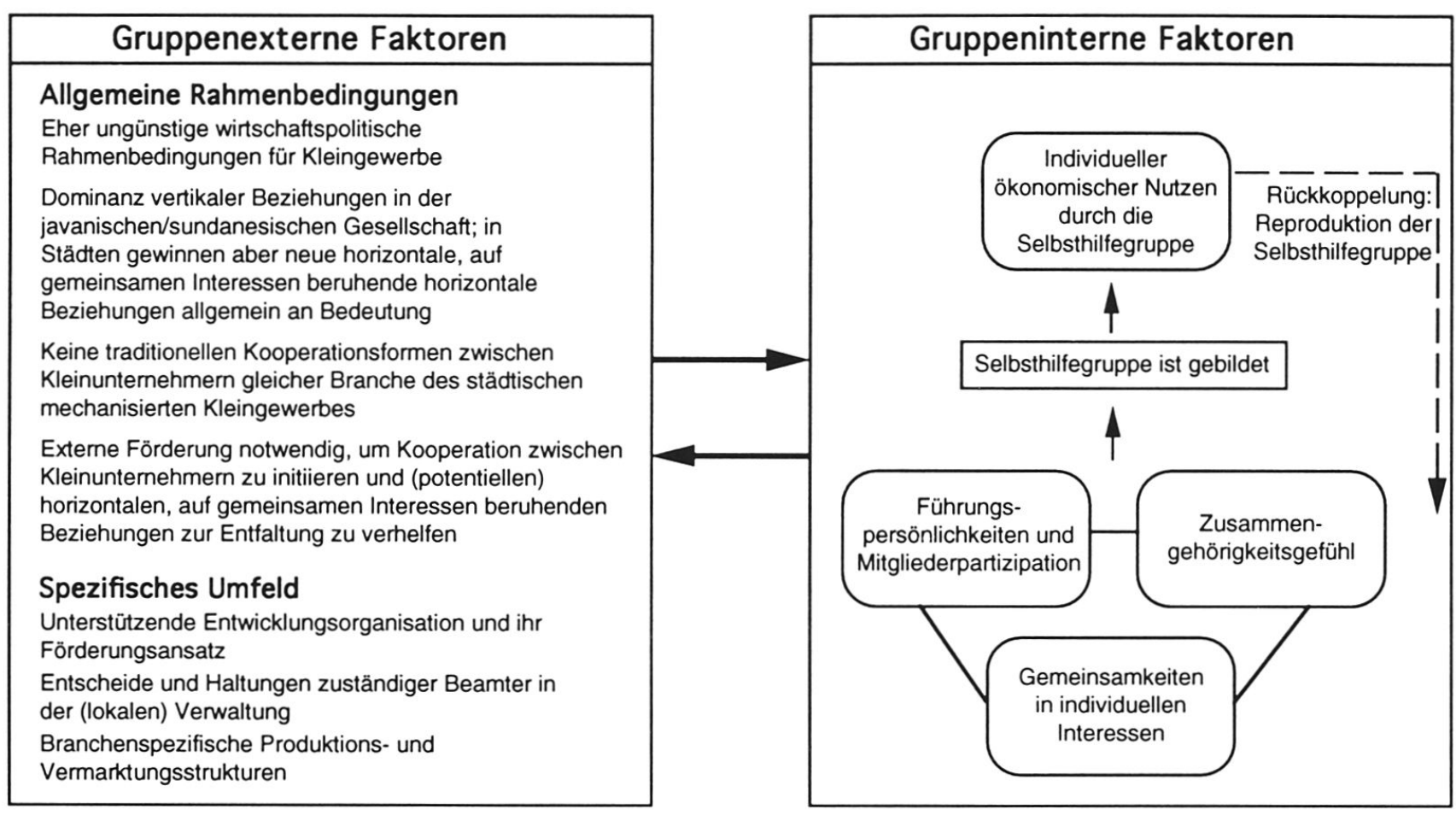

Abb.2 Gruppenexterne und -interne Faktoren für die Konstituierung von Kleinunternehmer-Selbsthilfegruppen im städtischen Java.

Möbelstücke und Baumaterialien, wie z. B. Tragbalken oder Fensterrahmen. Die Holzverarbeitung geschieht vorwiegend maschinell. Abnehmer sind Endverbraucher, Einzel- und Zwischenhändler (vgl. SCHMIDT 1988: 279).

\section{Anfangsphase}

1987 schlossen sich 40 Schreinermeister aus diesem Gebiet zu einer informellen Selbsthilfegruppe zusammen, um die Konkurrenz und den Preiskrieg untereinander zu lindern. Wenig später unterstützten ein indonesisches Managementinstitut und eine deutsche Parteistiftung diese Kleinunternehmer in betriebswirtschaftlichen Bereichen. Einige Schreiner wurden auch im Verbandsmanagement geschult und als Selbsthilfegruppenleiter ausgebildet. 1989 ließen sich die Schreiner als Genossenschaft unter dem Namen KIPKAB (Koperasi Industri Pengolahan Kayu dan Bahan Bangunan = Genossenschaft der Holzverarbeitungs- und Baumaterialienindustrie) staatlich registrieren.

Die erfolgreiche Gründung von KIPKAB läßt sich auf die spezifische Konstellation gruppenexterner und -interner Faktoren zurückführen. Die gruppeninterne Ausgangssituation war insofern günstig, als die Schreiner das Ziel gemeinsam hatten, die Preiskonkurrenz untereinander zu lindern sowie später den Einkauf durch Kooperation zu verbilligen. Dieses gemeinsame Interesse wiederum wurde durch das spezifische betriebswirtschaftliche Um- feld (verstärkte Konkurrenzsituation in der Holzbranche) geweckt. Später trug auch die externe Förderung, insbesondere die Ausbildung von Gruppenleitern sowie ferner auch die fremdorganisierten Trainings und Gruppentreffen, wesentlich zu verbesserten gruppeninternen Strukturen bei.

\section{Aufbauphase und anschließendes Ruhestadium}

Nach der Gründung von KIPKAB wurde der geplante gemeinsame Einkauf von Holz und Hilfsstoffen wie Leim, Nägeln oder Scharnieren in die Wege geleitet. Man beabsichtigte, das Rohmaterial zu einem günstigen Zeitpunkt zu erwerben und von Preisreduktionen für Massenlieferungen zu profitieren. Da ein solcher gemeinsamer Einkauf gruppeneigenes Kapital voraussetzt, baute KIPKAB auch eine Spar/Leih-Gruppe bzw. eine Kreditgenossenschaft auf. Dabei wurde sie von den unterstützenden Entwicklungsorganisationen finanziell und technisch unterstützt. In diese Kreditgenossenschaft mußten die Schreiner Pflichtbeiträge einzahlen; im Gegenzug konnten sie daraus zinsgünstige Darlehen beziehen. Kurz nach dem Aufbau einer Kreditabteilung mißbrauchten jedoch zwei Schreiner ihren Kredit und weigerten sich, diesen zurückzubezahlen. Erst auf Druck der beiden unterstützenden Entwicklungsorganisationen wurden die Darlehen doch noch zurückbezahlt. Trotzdem hatte dieses Ereignis einen Vertrauensschaden innerhalb der Selbsthilfegruppe zur Folge. Die Mitglieder zogen ihre Geldbeträge 
aus der Genossenschaftskasse zurück, so daß der geplante gemeinsame Einkauf nie realisiert werden konnte. So führte $K I P K A B$ lange Zeit keine Aktivitäten durch und galt bei den unterstützenden Entwicklungsorganisationen als Mißerfolg.

Dieser Mißerfolg von KIPKAB läßt sich damit erklären, daß gruppeninterne soziale Beziehungen und insbesondere das Zusammengehörigkeitsgefühl erst wenig entwickelt waren. So konnte beispielsweise den Kreditmißbräuchen nicht durch soziale Kontrolle vorgebeugt werden. Dieses Manko konnte langfristig auch nicht durch den Einfluß der unterstützenden Entwicklungsorganisationen wettgemacht werden. Wegen des vorzeitigen Scheiterns eines gemeinsamen Einkaufssystems sah auch niemand mehr einen individuellen ökonomischen Nutzen in der aktiven Mitgliedschaft bei KIPKAB. Dies ist wohl der Hauptgrund dafür, daß die Selbsthilfegruppe nicht mehr durch gruppenbezogenes Handeln der Kleinunternehmer reproduziert wurde.

\section{Reaktivierungsphase}

Zur Zeit der Untersuchung, Ende 1991, war KIPKAB wieder äußerst aktiv. Der auslösende Grund dafür lag nicht innerhalb der Selbsthilfegruppe, sondern bei einem Beschluß der Stadtregierung von Bandung, angrenzend zur Straße, wo die Schreinereien liegen, einen Stadtpark zu errichten und gleichzeitig die Straße zu verbreitern. Dazu sollten die Schreinerwerkstätten und die Wohnhäuser der Kleinunternehmerfamilien, welche in diesem Gebiet teilweise bereits seit 30 Jahren angesiedelt waren (sowohl auf eigenem Land als auch auf Land im Stadtbesitz), geräumt und abgebrochen werden. Die Stadtregierung hatte den Kleinunternehmern eine Entschädigung für ihre Betriebsgebäude und ihr Land offeriert. Diese war jedoch äußerst gering. Außerdem hatte die Stadt den Schreinern einen neuen Standort angeboten. Die Kosten für die Betriebsflächen am neuen Ort lagen jedoch außerhalb der finanziellen Möglichkeiten der Kleinunternehmer. Die Räumung der Schreinereien stand kurz bevor. Von der geplanten Umsiedlung waren praktisch alle Schreiner dieses Gebiets betroffen und in ihrer wirtschaftlichen Existenz bedroht. In der Folge reaktivierten die Schreiner ihre Selbsthilfegruppe in einer relativ kurzen Zeitspanne und traten mit der Stadtregierung in Kontakt, um einen erschwinglichen Preis für den angebotenen Ersatzstandort oder eine höhere Entschädigung für die bisherigen Betriebsgebäude auszuhandeln. In diesen Verhandlungen wurde $K I P K A B$ von den erwähnten Entwicklungsorganisationen aktiv unterstützt. ${ }^{13}$

Das Beispiel von KIPKAB illustriert, wie externer Druck in hohe Konvergenz der Interessen unter Kleinunternehmern und in der Folge in gemeinsame Aktivitäten münden kann. Unter solchen Umständen sind Individuen rascher willens, sich zusammenzuschließen, um unerwünschte Prozesse gemeinsam und wirkungsvoller abzuwenden. Dies gilt um so mehr, wenn die eigene Existenz gefährdet ist. Das Beispiel zeigt, daß selbst solche Selbsthilfegruppen, die unter normalen Bedingungen nicht er- folgreich arbeiten, dann wichtige Funktionen übernehmen können, wenn dies Rahmenzwänge erfordern. Ein positiver gruppenexterner Faktor für die Reaktivierung von KIPKAB liegt in der Unterstützung durch die zwei erwähnten Entwicklungsorganisationen. Denn ohne deren Beistand hätten die Schreiner wohl keine Verhandlungen mit der Stadtregierung einleiten können. Daß KIPKAB in einer solch kurzen Zeit reaktiviert werden konnte und so rasch aktionsfähig war, läßt sich aber nicht ausschließlich mit gruppenexternen Faktoren erklären. Vielmehr erwiesen sich dafür bereits einmal aufgebaute gruppeninterne Organisationsstrukturen sowie früher bestimmte und ausgebildete Führungspersönlichkeiten als nützlich. Ferner verstärkte sich durch die erneute Aufnahme von Gruppenaktivitäten das Zusammengehörigkeitsgefühl innerhalb der Selbsthilfegruppe.

\section{Schlußfolgerungen}

Die Konstellation gruppeninterner und -externer Kräfte ist ausschlaggebend für die Konstituierung einer Selbsthilfegruppe und bewirkt auch Schwankungen in der Intensität von Gruppenaktivitäten. So können Ruhephasen z. B. mit gruppeninternen Faktoren erklärt werden wie der Verschlechterung sozialer Beziehungen unter den Mitgliedern oder der Unfähigkeit und Erfolglosigkeit der Gruppe oder ihrer Leitung bezüglich der Zielerreichung. Aber Gruppenaktivitäten können auch deshalb ruhen, weil die Mitglieder aufgrund gruppenexterner Gegebenheiten den Zusammenschluß über längere Perioden gar nicht benötigen (z. B. weil gerade durch die Selbsthilfegruppe ein kurzfristiges Ziel erreicht und/ oder das Umfeld verbessert worden ist). Bei auftretendem Bedarf ist aber eine ehemals vorhandene gruppeninterne Struktur schneller reaktivierbar bzw. eine Selbsthilfegruppe rascher handlungsfähig, als eine gänzlich neue Selbsthilfegruppe aufgebaut wäre. Jedenfalls greifen diejenigen Erklärungsansätze zu kurz, welche das erfolgreiche Entstehen und Fortbestehen von Selbsthilfegruppen entweder allein auf eine "gute Gruppe» oder ausschließlich auf eine "Reaktion aufäußere Bedingungen» zurückführen.

Kleinunternehmer können durch kollektives Handeln in einer Selbsthilfegruppe besser Einfluß auf ihr spezifisches Umfeld (kaum aber auf die allgemeineren Rahmenbedingungen) nehmen. Umgekehrt aber kann gemeinsames Handeln von Rahmenbedingungen beeinflußt und strukturiert und durch das spezifische Umfeld ausgelöst werden. Dabei spielen Entwicklungsorganisationen eine wichtige Rolle. Denn KleinunternehmerSelbsthilfegruppen werden oft von Entwicklungsorganisationen initiiert und bleiben paradoxerweise auch längerfristig auf deren Unterstützung angewiesen. So stellt sich schließlich die weiterführende Frage, ob so nicht auch neue Abhängigkeitsbeziehungen entstehen. 
Anmerkungen

1 Der vorliegende Artikel basiert auf Teilen der am Geographischen Institut der Universität Zürich durchgeführten Diplomarbeit des Autors, "Kleinunternehmer-Selbsthilfegruppen im städtischen Java, Indonesien: Rahmenbedingungen und Konstituierung von Selbsthilfegruppen im mechanisierten Kleingewerbe" (1993). Die Daten wurden während eines viermonatigen Feldaufenthalts im Rahmen eines studentischen Praktikums mit Swisscontact, Schweizerische Stiftung für technische Entwicklungszusammenarbeit, auf Java, Indonesien, Ende 1991 gesammelt. Dabei wurden gelenkte, qualitative Interviews mit Repräsentanten der 15 untersuchten Selbsthilfegruppen, freie Befragungen von 25 Fachleuten der Entwicklungszusammenarbeit in Indonesien (indonesische und ausländische Nicht-Regierungsorganisationen, kirchliche, universitäre und staatliche Stellen) und einige Werkstattbesuche im mechanisierten Kleingewerbe durchgeführt.

2 Vgl. z. B. SCHILD (1993).

3 Vgl. beispielsweise WÄLTY et al. (1990).

${ }^{4}$ Für Indonesien beispielsweise ELDRIDGE (1989), FRINGS (1991), GUINAND (1992), HOLLOWAY (1989), UTRECHT (1986).

${ }^{5}$ Selbsthilfegruppen im ländlichen Java werden beispielsweise von BONGARTZ (1987) thematisiert.

${ }^{6}$ Zahlenangaben im obigen Absatz basieren auf FISCHER WELTALMANACH 1993.

${ }^{7}$ Beim Begriff Kleinunternehmer-Selbsthilfegruppen wird deshalb nur die maskuline Form aufgeführt, weil die untersuchten Gruppen ausschließlich aus Männern bestehen.

${ }^{8}$ Eine Ausnahme bilden Kleinunternehmer chinesischer Abstammung, die auch geschäftlich innerhalb inrer Familienclans häufig und intensiv kooperieren. Die später eingewanderte chinesische Bevölkerungsgruppe bildet in Indonesien eine Minderheit zur "einheimischen" Bevölkerung, den sog. pribumi.

9 Extern initiierter Aufbau und Unterstützung von Selbsthilfegruppen werden im Entwicklungsjargon "Selbsthilfeförderung" genannt. Zum entwicklungstheoretischen und - praktischen Konzept der Selbsthilfeförderung vgl. VERHAGEN (1987), MÜNKNER (1989) und VERON (1993: 9 ff.).

10 Arisan kann als "Gemeinschafts-Sparverein" definiert werden, in dem die Mitglieder regelmäßig (z. B. wöchentlich) eine bestimmte Summe Geld in eine Gemeinschaftskasse einzahlen. Die Gesamtsumme der eingelegten Mittel steht dem Mitglied zu, das gemäß einem festgelegten Rotationssystem zum Ziehen berechtigt ist. In Ausnahmesituationen (wie z. B. anfallenden Arztkosten) können die Spareinlagen außerterminlich in Anspruch genommen werden. Arisan erleichtert es Personen, auf ein bestimmtes Ziel hin eine gröBere Summe zu sparen (HARDJONO 1987: $240 \mathrm{f}$.)

11 Im Kleingewerbe sind auch in indonesischen Städten durchaus ohne externe Unterstützung entstandene wirtschaftliche Kooperationsformen vorhanden. So existieren in Jakarta beispielsweise Gruppen von Migranten aus gleichen Herkunftsdörfern, welche soziale und ökonomische Netzwerke bilden (vgl. EVERS 1989: $161 \mathrm{ff}$.). Diese Gruppen setzen sich aber nicht aus selbständigen Kleinunternehmern zusammen.

12 Die angegebene Bevölkerungszahl von NUDS (1985) beruht auf einer funktionalen und nicht auf der offiziellen administrativen Abgrenzung der Stadt Bandung.
13 Der endgültige Ausgang der Verhandlungen mit der Stadtregierung und deren Auswirkungen auf das Fortbestehen von KIPKAB sind dem Autor leider nicht bekannt.

\section{Literatur}

BONGARTZ, H. (1987): Prospects and Limitations of SelfHelp Organizations in the Development Process of Rural Java: A Case Study on "Usaha Bersama" Groups in the Special Province of Yogyakarta, Indonesia, Bielefeld.

ELDRIDGE, P.(1989): NGOs in Indonesia: Popular Movement or Arm of Government?, Clayton.

EVERS, H.-D. (1989): Urban Poverty and Labour Supply Strategies in Jakarta. In: RODGERS, G. (ed.): Urban Poverty and the Labour Market: Acces to Jobs and Incomes in Asian and Latin Armerican Cities, 145-172, Geneva.

FISCHER WELTALMANACH 1993, Frankfurt a. M

FRINGS, U. (1991): Rolle und Funktion von nichtstaatlichen Organisationen in Indonesien, Hamburg.

GUINAND, Y. (1992): Möglichkeiten und Grenzen der Selbsthilfeförderung in Indonesien. In: Geographica Helvetica, Nr. 2, 71-80

HARDJONO, J. (1987): Land, Labour and Livelihood: In a West Java Village, Yogyakarta.

HILL, H. (1992): Survey of Recent Developments. In: Bulletin of Indonesian Economic Studies, Vol. 28, No. 2, 3-41.

HOLLOWAY, R. (ed.) (1989): Doing Development: Governments, NGOs and the Rural Poor in Asia, London.

LEEMANN, A. (1993): Einleitung zum Themenheft Bali (Indonesien). In: Geographica Helvetica, Nr. 1, 3-5.

MULDER, N. (1990): Individuum und Gesellschaft in Java: Eine Untersuchung zur kulturellen Dynamik, Saarbrücken/ Fort Lauderdale.

MÜNKNER, H. (ed.) (1989): Entwicklungspolitische Konsequenzen einer konsequenten Selbsthilfeförderung, Marburg.

NUDS (NATIONAL URBAN DEVELOPMENT STRATEGY) (1985): Urban Growth and Structure in Indonesia. Report T1.6/C3, Jakarta

SCHILD, A. (1993): Außenpolitik und Zivilgesellschaft in der Schweiz: Ein Essai zur schweizerischen Entwicklungszusammenarbeit, Bern.

SCHMIDT, F. (1988): Der Beitrag des städtischen informellen Sektors zur sozialökonomischen Entwicklung Indonesiens: dargestellt am Beispiel der Stadt Bandung, Berlin.

UTRECHT, A. (1986): NGOs in Indonesia: Some Comments on Their Role in the Process of Social Change. In: Kabar Seberang, No. 17, 199-210.

VERHAGEN, K. (1987): Self-Help Promotion: A Challenge to the NGO Community, Oegstgeest/Amsterdam

VERON, R.: Kleinunternehmer-Selbsthilfegruppen im städtischen Java, Indonesien: Rahmenbedingungen und Konstituierung von Selbsthilfegruppen im mechanisierten Kleingewerbe, Diplomarbeit am Geographischen Institut der Universität Zürich.

WÄLTY, S., et al. (eds.) (1990): Von nachholender zu nachhaltiger Entwicklung: Beiträge zur Entwicklungsforschung, Anthropogeographie Universität Zürich, Vol. 10. 